\title{
Interculturalité et santé des personnes originaires de pays d'Afrique subsaharienne en France
}

\author{
Gildas Vieira ${ }^{12}$, Robert Courtois ${ }^{34}$, Emmanuel Rusch $^{2}$,
}

${ }^{1}$ Fédération Régionale des Acteurs en Promotion de la Santé (FRAPS), 37000 Tours, France

${ }^{2}$ CHRU de Tours, Département de Santé Publique et d'Information médicale, Equipe 'Education Ethique Santé', 37044 Tours cedex 9, France

${ }^{3}$ Université François Rabelais de Tours, PRES Centre-Val de Loire Université, Département de psychologie, EA 2114 'Psychologie des âges de la vie et adaptation', 37041 Tours cedex, France

${ }^{4}$ CHRU de Tours, Clinique Psychiatrique Universitaire, 37044 Tours cedex 9, France 


\section{Résumé}

La démarche communautaire en santé permet d'agir sur les inégalités de santé, nous avons souhaité la développer auprès de populations originaires d'Afrique subsaharienne en France. Nous avons mesuré les effets sur les comportements de santé sous l'angle des rapports interculturels.

Cette démarche repose sur une méthodologie constituée à la fois d'outils de recherche en psychologie, de protocole d'intervention en santé publique et d'un focus group sur plusieurs séances d'échanges, au cours duquel nous prêterons une attention particulière aux relations interculturelles. Il s'agit d'une démarche de santé communautaire, elle fait partie intégrante de la santé publique en constituant une stratégie au sein des démarches de promotion de la santé. Ce travail montre que cette démarche est un outil pertinent d'analyse de comportement, il favorise par ailleurs l'amélioration des aptitudes en promotion de la santé des participants.

The community approach in health makes it possible to act on inequalities of health, we wished to develop it with populations originating from sub-Saharan Africa in France. We measured the effects on health behaviors in terms of intercultural relationships.

This approach is based on a methodology consisting of both research tools in psychology, protocol intervention in public health and a focus group on several exchange sessions, during which we will pay particular attention to intercultural relations. It is a community health approach, it is an integral part of public health by being a strategy in the process of health promotion. This work shows that this approach is a relevant tool of behavior analysis, it also promotes the improvement of participants' health promotion skills.

\section{Entrées d'index}

Mots clés : santé communautaire, inégalité sociale en santé, communauté d'Afrique subsaharienne, interculturalité.

Key words : community health, social inequality in health, community of sub-saharan Africa, interculturality.

\section{Plan}

Introduction

Renforcer l'action communautaire pour un plus grand contrôle sur sa santé

Construire une démarche de santé communautaire participative

Résultats et discussion autour des concepts interculturels

L'incidence du regard social sur les maladies chroniques

Représentation de la santé et de la maladie dans l'interculturalité

Des pistes d'amélioration du niveau de santé dans le cadre d'un processus d'empowerment

Conclusion 


\section{Introduction}

La démarche communautaire en santé (DCS) se définit comme un «ensemble de processus permettant aux gens, individuellement ou collectivement, d'augmenter leur contrôle sur leur santé et sur ses déterminants » (O'Neill, 2012: p. 67-69). Elle vise à réduire les inégalités sociales de santé en favorisant un travail sur les représentations et l'intermédiation interculturelle (Legros \& Olivera, 2011 : p. 172-178) dans le sens de l'amélioration de la communication entre les institutions et dans notre contexte auprès des familles africaines. Sa spécificité est d'être collective et non individuelle et de promouvoir une vision globale et de proximité. La dimension éducation pour la santé est intégrée dans cette démarche avec la recherche de l'empowerment (Augoyard \& Renaud, 1998: p. 28-35) du groupe, un processus de prise de conscience de sa situation et des possibilités d'action associées au développement personnel et communautaire de ses ressources de vie et de santé.

Les séances de DCS sous forme de focus group permettent d'identifier les représentations de la santé, des habitants et des professionnels de santé dans un territoire défini. Nous nous intéresserons aux communautés d'Afrique subsaharienne (AFS) au sein des zones urbaines en région Centre-Val de Loire en France ; ces populations ont, bien entendu, des identités culturelles différentes, mais partagent des conceptions et une compréhension proche en matière de santé, car elles sont issues de sociétés traditionnelles (Tilmans-Cabiaux \& Mokrane \& Ravez, 2013). La santé est appréhendée dans une acception positive et globale comme le définit l'Organisation Mondiale de la Santé (OMS), une vision de la Santé qui inclut pour les populations d'Afrique subsaharienne (AFS) des références culturelles à un ensemble de notions beaucoup plus diverses, tels que la bénédiction, la punition divine, ou encore l'ensorcellement (Fainzang, 1981 : p. 21-415). Dès lors, la mise en œuvre des politiques de Promotion de la Santé (PS) doit prendre en compte ces spécificités et adapter les actions à ces particularités pour plus d'efficacité (Reinberg, 2014: p.75-84). En effet l'interculturalité peut être définie comme l'ensemble des processus psychiques, relationnels, groupaux, et institutionnels, généré par les interactions des cultures, dans un rapport d'échanges réciproques (Tilmans-Cabiaux et al, 2013).

Les populations d'AFS sont importantes et en nombre croissant en France (Lessault \& Beauchemin, 2009 : p.163-194), ces dernières sont regroupées pour grande partie dans des quartiers dits sensibles, souvent défavorisés en termes d'inégalité sociale et de santé (Carde, 2014). Nos travaux sur les déterminants comportementaux de santé, sous forme de recherche interventionnelle en santé des populations (RISP), permettent d'utiliser des méthodes scientifiques pour un impact favorable sur les déterminants sociaux, culturels et environnementaux.

L'article a pour objet d'identifier les conditions de réussite d'une DCS menée sous forme de focus group (Baribeau \& Germain, 2010: p. 28-49) prenant en compte l'interculturalité. Cette étude appréhende principalement l'impact sur l'amélioration des compétences sanitaires de cette population d'origine migrante ${ }^{1}$, et la réduction de certains facteurs spécifiques pouvant affecter leur santé.

\section{Renforcer l'action communautaire pour un plus grand contrôle sur sa santé}

Ce travail s'appuie sur les principes de la charte d'Ottawa afin de renforcer « la participation active et concrète de la communauté à la fixation des priorités, à la prise de décision...pour atteindre un meilleur niveau de santé $»^{2}$. En effet, le système de santé compte pour 12 à $20 \%$ sur l'état de santé d'une population, les $80 \%$ restant se jouent hors du système de santé (Center for Disease Control and Prévention, 1982). La santé est influencée par des facteurs et déterminants sociaux, environnementaux, économiques. La DCS est un instrument approprié permettant d'apprécier ces impacts et d'agir dans un second temps sur les déterminants de santé. L'efficacité d'un cette démarche devrait, en théorie, se mesurer à partir des modifications des déterminants de santé de la population cible, ou mieux, des indicateurs de santé résultant des recommandations mises en œuvre. Il est en général assez difficile de

1

Selon la définition adoptée par le Haut Conseil à l'Intégration, le migrant appelé aussi l'immigré est une personne née étrangère à l'étranger et résidant en France. Les personnes nées françaises à l'étranger et vivant en France ne sont donc pas comptabilisées.

2

La Charte d'Ottawa pour la promotion de la santé a été établie à l'issue de la première Conférence internationale sur la promotion de la santé, Ottawa (Canada), du 17 au 21 novembre 1986. Elle énumère 5 axes dont l'axe 3 Renforcer l'action communautaire. 
pouvoir évaluer de tels changements du fait de la multiplicité de facteurs extérieurs au projet pouvant intervenir sur ces évolutions de l'état de santé des publics potentiellement impactés. L'efficacité d'une DCS se mesure plus couramment à partir des changements opérés du fait du processus, allant de la prise en compte et la mise en œuvre des recommandations à des effets plus généraux comme l'implication dans la vie locale en matière de santé.

La DCS peut être un outil d'aide à la décision. Des recommandations sont proposées afin de maximiser les impacts positifs pour la santé et de minimiser les impacts négatifs.

\section{Constitution du groupe de santé communautaire et planification du focus group}

Nous avons entrepris entre avril et juillet 2013 des entretiens en région Centre-val de Loire, en allant vers des personnes originaires de pays d'AFS, des entrevues de 30 minutes basées sur de la sensibilisation à la promotion de la santé. Ces temps nous ont permis de nous familiariser aux spécificités en santé de cette population. Ces entretiens se terminaient par l'invitation à participer à un groupe de santé communautaire.

Parmi les personnes interrogées, nous recevrons 16 retours positifs, 10 hommes et 6 femmes. Nous avons mené dix séances avec ces derniers de septembre 2013 à juin 2014, à raison d'une séance mensuelle, chaque premier samedi du mois. Trois sessions avec uniquement les membres de la communauté africaine et deux animateurs dont un membre moteur de cette communauté, volontaire et intéressé par ces questions, et moi-même étudiant chercheur ayant vécu plusieurs années en Afrique. Nous avons pris un temps plus important avec le co-animateur, de formation en coaching, pour une sensibilisation à la promotion de la santé, et la conduite d'une démarche communautaire en focus group. Nous souhaitions créer un climat de confiance avec des règles d'échanges fixées par le groupe allant de la confidentialité à la libre prise de parole sans être interrompu ; puis dans un second temps, intégrer des professionnels de santé et/ou des acteurs de la politique dans les échanges.

Nos objectifs étaient de se questionner sur les comportements en santé, comprendre le fonctionnement des politiques publiques territoriales, travailler sur l'empowerment en santé, identifier les problèmes et les besoins en santé liés à la communauté et définir des actions prioritaires. Cela a conduit à réfléchir à la manière dont on pouvait y répondre en prenant en compte les spécificités de la population et l'interculturalité. Nous avions établi un rétro planning avec une rencontre tous les premiers samedis du mois de $13 \mathrm{~h}$ à $15 \mathrm{~h}$ et un ordre du jour répondant à nos attentes.

\section{Construire une démarche de santé communautaire participative}

La population visée est constituée de personnes issues de communautés d'AFS en France âgée de 18 ans et plus résidentes à Blois (département du Loir-et-Cher en région Centre-Val de Loire), l'âge moyen était de 44,69 ans. Les tranches d'âges des personnes interviewées, quelle que soit leur catégorie socio-professionnelle (CSP) et leur niveau d'étude, variaient pour les femmes entre 29 et 62 ans et les hommes entre 26 et 65 ans. Les répondants ont été rencontrés au hasard dans les quartiers prioritaires de la région Centre-Val de Loire en France. Parmi les 16 répondants du focus group, on retrouve 6 femmes et 10 hommes, principalement d'origine Guinéenne, Congolaise et Malienne. Le niveau d'étude est post bac pour 6 personnes du groupe, 7 personnes sans emploi, 5 avec des emplois précaires et 11 des 16 ne sont pas imposables. Après les 3 premières séances, 2 professionnels de santé du territoire et 2 personnalités politiques seront invités à ces travaux. Nous avons construit cette RISP (Ferron \& Breton, 2013 : p. 425) en progressant dans le processus tel que décrit ci-après.

Nous avons sollicité des personnes au hasard en allant dans les quartiers prioritaires de la région Centre-Val de Loire, Les populations des quartiers sont, plus que d'autres, victimes d'un état de santé dégradé, aggravé par un accès aux soins de premier recours défaillant. Nous avons proposé un entretien qualitatif sur trois grands points : leur parcours, leur connaissance en PS, leur perception des inégalités sociales en santé et le souci d'améliorer les comportements, avant de les inviter à participer à un focus group sur 10 mois. Afin de satisfaire à la phase de planification, des prérequis semblaient indispensables pour réussir cette démarche. Nous avons exploré dans un premier temps la littérature et la recherche sur la relation de cette population à la PS. Dans un second temps, des voyages au Congo, Sénégal, Mali et en Guinée, ont permis une immersion des deux animateurs, deux hommes, dans les réalités des populations de ces pays. L'un des deux étant d'origine d'AFS, l'autre d'origine française, l'effet enquêteur peut se questionner, la présence des deux animateurs durant les séances pouvait toutefois garantir une certaine pertinence des réponses et une authenticité des discussions. Les 
conditions ci-avant réunies, la recherche interventionnelle en France pouvait dès lors se réaliser.

Nous avons mesuré le travail accompli et analyser les retours du groupe. Une évaluation à chaud est réalisée à l'issue de cette démarche, puis une évaluation à distance à 12 mois. Nous nous sommes intéressés notamment aux relations interculturelles et à l'amélioration des comportements de promotion de la santé (Filiatrault \& Richard, 2005 : p. 45-56).

\section{Résultats et discussion autour des concepts interculturels}

Les échanges ont fait ressortir 4 concepts et thématiques liées à l'interculturalité :

a- La compétence transculturelle ;

b- L'interprétariat communautaire ;

c- Le «mainstreaming » migration ;

d- La Littératie en santé.

a) La compétence transculturelle

Les participants ont évoqué la capacité à reconnaître et à comprendre des conceptions du monde différent et à en tenir compte dans son action (Archinard \& Berthod, 2014). Les différences de références (éthique, sociale, culturelle et religieuse) au sein même du groupe sont notables dans l'expression car nous avions des personnes originaires de Guinée, du Congo, principalement, et une personne du Togo, du Sénégal et du Mali. Le recours aux soins devrait tenir compte de leurs spécificités, et des niveaux de santé inégaux arguait notamment les femmes du groupe assez solidaires dans les échanges et sur leurs points de vue. En effet, dans ce groupe multiculturel, les points communs sont nombreux car ils sont originaires de pays de culture traditionnelle. La compréhension de la notion de santé reste, somme toute, identique, avec une définition plutôt biomédicale, et la présence divine couvrant cette notion avec la répétition par les personnes d'Afrique de l'Ouest du mot : «Inch Allah » signifiant s'il plait à Dieu. Chaque participant parlait français de manière compréhensive par tous, nous n'avons pas eu besoin de traduction, sinon parfois des reformulations des femmes guinéennes au nombre de trois, en Soussou ou Malinké, des dialectes Guinéens.

b) L'interprétariat communautaire

Il permet la communication entre des interlocuteurs de langues et de cultures différentes, c'est un outil indispensable dans le «aller vers» en matière de santé. Le groupe pense que des référents communautaires sont indispensables. Au sein même du groupe le partage de l'information nécessite parfois une réinterprétation ou reformulation pour s'adapter aux facultés de compréhension de chacun. Plusieurs personnes évoquent que les hôpitaux devraient avoir des relais communautaires pour permettre une meilleure prise en charge des migrants. L'une des participantes rapporte que «les personnes relais ou femmes relais faisaient en partie ce travail d'accompagnement. Aujourd'hui ce n'est plus le cas, cette association a disparu, la question se pose donc à Blois qui connaît une croissance de migrants et primo-arrivants depuis plusieurs années ».

c) Le « mainstreaming » migration

Il s'agit d'intégrer la notion migratoire et communautaire dans les domaines de l'information, de la communication, et de la mise en réseau. Le groupe était en demande d'amener les acteurs de la politique, de l'administration et de la société à inclure la dimension migratoire dans leur réflexion et leur action, aux niveaux de la planification, de la mise en œuvre et de l'évaluation des projets auxquels ils participent.

d) La Littératie en santé

Nos discussions autour de ce concept ont été très animées, nous avons également largement abordé l'interculturalité, l'accès à l'information et l'illettrisme (Rootman et al, 2006: p. 87). Selon l'Organisation Mondiale de la Santé (OMS) : «La littératie en santé est la capacité de trouver, de comprendre, d'évaluer et de communiquer l'information de manière à promouvoir, à maintenir et à améliorer sa santé dans divers milieux au cours de la vie... Étant donné que les compétences en matière de santé améliorent l'accès des individus à des informations sur la santé et leur capacité à les utiliser efficacement, elles sont essentielles pour l'obtention de moyens d'agir [...]» (Collette \& Rousseau, 2013 : p. 107-131).

Le débat a été constructif, il a permis une réflexion sur le lien alphabétisation/analphabétisme et santé dans différents domaines comme la promotion de la santé mais également l'éducation pour la santé et la communication/information en santé. Un homme du groupe souligne que le défaut de compréhension de certains codes dans le domaine de la promotion de la santé en France ne doit pas 
s'interpréter comme un manque d'intelligence de certaines personnes de culture différentes et qui sont parfois sur-diplômées dans leurs pays d'origine.

\section{L'incidence du regard social sur les maladies chroniques}

La thématique VIH et sexualité (Hall \& Hacker, 1984) a souvent été abordée lors des séances, avec une crainte de stigmatisation des personnes de la communauté sur ce sujet sensible. La question du Sex Dry (Sexe sec) qui oblige certaines femmes d'AFS à user de pratique sexuelle avec le vagin sec afin de satisfaire voire garder leurs compagnons fut une découverte pour un grand nombre. Les hommes du groupe notamment s'étonnaient que ces pratiques favorisent la contamination des maladies sexuellement transmissibles.

En effet un membre relate l'effet pervers des politiques de promotion de la santé lorsque l'on ne connaît pas sa culture, par exemple une expérience de distribution de préservatifs en action de prévention dans des lieux de présence de la communauté, conduit les femmes à penser qu'on incite leurs maris à l'infidélité. L'information sur les Trods (Test Rapide d'Orientation Diagnostique pour le Sida) qui permettent un dépistage moderne, anonyme et rapide auprès d'associations habilitées, est une évolution sur la maladie qui a été débattue et bien accueillie.

Un travail sur la communication et l'implication des communautés dans les actions de PS autour de la maladie leur a semblé nécessaire (Marsicano et al, 2014 : p. 516). Aborder la question du VIH ne veut pas dire seulement relever les tabous ou pointer la communauté africaine comme forte porteuse de la maladie, simplement sur la base des chiffres qui souligne que $2 / 3$ des personnes touchées dans le monde sont en AFS : 26 millions sur 36 millions. Une réflexion sur d'autres prises en charge touchant le patient migrant comme le diabète par exemple est à explorer avec des réseaux de proximité existants.

Plusieurs maladies infectieuses et chroniques présentent de fortes prévalences chez les migrants, notamment la drépanocytose, la tuberculose, le VIH, les hépatites virales et les cancers du foie (Marsicano et al, 2011 : p. 313-341). Les politiques publiques ont réfléchi pour interagir de façon globale sur la population, mais elles doivent prendre en compte ces spécificités communautaires. En effet, à l'image de cette femme du groupe qui explique par exemple ne pas souhaiter être aperçue dans une salle d'attente de médecin, ou accompagnée de professionnels travaillant sur le VIH ou le dépistage de Cancer de peur que l'on s'imagine qu'ils ont une maladie grave. Elle craint comme elle le dit que « certains compatriotes s'empressent d'annoncer des informations fausses à la famille restée au pays » ou d'être stigmatisée.

Quelques points de la démarche sont cependant à améliorer. En effet lors de cette démarche, nous aurions pu prévoir plus de temps sur la compréhension de l'approche communautaire et la PS. Les délais et fréquence de travail peuvent être revus en fonction des besoins des participants. Nous avons connu 3 séances avec 8 personnes, peut être qu'un intéressement aurait aidé à la mobilisation. Nous nous questionnons sur le caractère transposable et pérenne de la démarche pour toute communauté, en effet une démarche d'évaluation d'impact en santé sur une thématique choisie par les habitants serait une suite intéressante à ce projet.

\section{Représentation de la santé et de la maladie dans l'interculturalité}

L'interculturalité semble avoir clairement un impact sur les comportements dans le domaine de la promotion de la santé des personnes du groupe qui sont de différentes cultures. Le phénomène de multiculturalité ne désigne pas uniquement un phénomène lié aux migrations mais ce qui réunit des individus aux normes, aux valeurs, aux représentations différentes. Se confronter et s'ouvrir aux autres, c'est accueillir de nouvelles perceptions, mais aussi prendre le risque de voir certaines de ses valeurs acquises remises en cause. Un médiateur culturel est cependant nécessaire à la gestion de situations interculturelles. Nous pouvons nous appuyer sur le schéma de Kohls, dit de «l'iceberg», afin de mieux comprendre les difficultés que nous avons à rencontrer l'autre au travers de sa culture (Albert, 2000 : p. 117).

La métaphore de l'iceberg met en relief la différence entre la partie visible et la partie invisible de l'iceberg, la seconde partie œuvrant pour la stabilité de l'iceberg, étant plus importante que la première. L'analogie à la communication interculturelle conduit à nous demander sur quelle base nous fondons nos perceptions et interprétations, lorsqu'une partie importante de notre propre iceberg nous est parfois ignorée, et celle de l'autre peut être invisible. Cela traduit la difficulté d'évaluer les 
attitudes et actions des autres. Les valeurs, d'après Stella Ting-Toomey (Ting-Toomey, 2012), spécialistes de la communication interculturelle, permettent d'interpréter et d'évaluer le comportement de personnes étrangères à notre culture. Plusieurs exemples de situations abordées reflètent les difficultés liées à l'interculturalité dans le soin où se confrontent différentes cultures, celles du patient, du soignant, de l'institution, et du système de santé :

- L'inobservance des traitements, le refus de soins

- Les pratiques religieuses ou rituelles d'un patient, d'un soignant

- La relation d'autorité au sein d'une équipe ou d'une famille

- La mise en œuvre de la laïcité à l'hôpital

- Rapports hommes/femmes

- Le rapport à l'espace, au temps, ponctualité d'un patient ou d'un soignant

Les apports d'Edward T. Hall, anthropologue américain, nous aident à comprendre ces questions d'espace et de temps (Hall \& Hacker, 1984). Ce dernier a étudié les habitudes et les perceptions que nous avons des autres peuples. Il explique que la notion du futur diffère suivant les cultures, et l'appréciation est très différente sur la notion de retard. Hall parle de sociétés monochrones et de sociétés polychrones.

La conduite de ce groupe de personnes d'Afrique de l'Ouest comme d'Afrique Centrale plutôt hétérogène, nous montre que les conduites ne sont pas uniquement le produit d'une culture d'origine. Il s'agit d'un ensemble de personnes aux trajectoires de migration différentes et singulière, avec une diversité de choix identitaires et de conduites face à la santé.

Le plus jeune homme du groupe évoquera que la culture d'origine est parfois jugée à tort, comme responsable de toutes les incompréhensions, et que ces populations ne seraient pas suffisamment actrices de leur santé. C'est sans prendre en compte dit-il la précarité, les logements indécents, le chômage qui touche ces personnes. Ce dernier âgé de 26 ans et en France depuis une quinzaine d'années estime que malgré une identité communautaire, l'intégration à la société de l'ensemble des populations migrantes se fait en France au travers des groupes professionnels, religieux, associatifs, politiques souvent multiculturels, modifiant ainsi les interprétations identitaires liées au pays d'origine. Son voisin âgé d'une quarantaine d'année et en France depuis 5 ans reprendra en disant que même en Afrique la mondialisation modifie rapidement les comportements tels qu'on les percevait et que la «pensée européenne gagne du terrain » au détriment des coutumes et traditions locales. Il explique par exemple que même la croyance à la sorcellerie ou aux guérisseurs s'estompe au profit de la médecine européenne, et que discuter de sorcelleries devient une manière simplement de s'identifier à un groupe en faisant semblant d'y croire encore. Il sera contredit par l'une des femmes originaire du Congo qui précise avec sérieux que «s'il y a moins d'actes de sorcelleries en France c'est parce que les mauvais sorts ne traversent pas l'Océan pour nous toucher en France ».

Le rapport à l'irréel reste toutefois bien présent dans la culture du soin lorsque la médecine ne trouve pas rapidement de solutions. Tout le groupe sera d'accord lorsque l'une des femmes évoque qu'en Afrique ils ne peuvent bénéficier de la protection sociale et la qualité de soins offerte en France, et que de nombreux décès sont plutôt liés à des difficultés de prise en charge ou de diagnostics plutôt qu'à la sorcellerie. Le regard de l'autre et l'opinion des personnes restées au pays restent très important et suscite des craintes.

\section{Des pistes d'amélioration du niveau de santé dans le cadre d'un processus}

\section{d'empowerment}

Un questionnaire d'évaluation à chaud a été remis aux participants lors de la dernière séance du focus group. On y trouve les réponses synthétisées ci-après :

La DCS a intéressé l'ensemble des participants. Elle a répondu aux attentes et aux objectifs fixés en début de démarche pour 13 participants, et 11 personnes pensent que la démarche a permis une évolution de leur comportement en faveur d'actions de promotion de la santé. Les participants à la DCS ont abordé, entre autres, deux grands sujets d'une part la promotion de la santé sous l'angle de l'interculturalité et d'autre part les maladies chroniques avec des échanges animés autours du VIH et de la Drépanocytose.

Le groupe a souhaité apporter quelques recommandations et propositions d'actions pour la communauté, dans un objectif de réduction des inégalités sociales en santé :

Euvrer pour la propreté dans les quartiers ; 
Un travail partenarial entre associations de migrants et les structures hospitalières pour l'amélioration de la prise en charge médico-sociale de ces personnes ;

Participer à la Démocratie local et aux conseils de quartiers ou conseils citoyens ;

Désigner des référents de santé communautaires pour l'orientation et l'accompagnement vers les soins ;

Proposer les membres du groupe comme des personnes ressources pour la population du quartier ;

La mixité femme/homme a permis une plus grande expression des femmes quel que soit leur pays d'origine, elles ont eu une prise de parole facile et solidaire entre elles de leurs propos, parfois face aux hommes notamment dans certains débats tels que leur position sur les barrières socio-économiques, la barrière de la langue, les différences culturelles, voire les discriminations comme autant de freins à l'accès aux soins. Les échanges étaient respectueux et courtois des points de vue. Ils ont valorisé le rôle des femmes dans l'éducation pour la santé dans le foyer, auprès des enfants et des autres femmes de la communauté.

L'évaluation à 12 mois après la fin des séances de groupe, confirme l'intérêt pour cette démarche, puisque 10 personnes sur les 16 de départ, poursuivent des actions de promotion de la santé alors qu'elles n'étaient pas engagées dans le type d'actions auparavant.

Cette étude ne saurait s'élargir à un continent africain pluriculturel, avec des représentations différentes de la santé, cependant malgré les différences culturelles et religieuses, les représentations de la santé et la compréhension de la problématique de la promotion de santé restent identiques. Les liens construits dans les lieux de vie que sont les quartiers dits sensibles leur permettent un espace d'échange commun libre et une mixité communautaire plus grande favorisant pour ces premières générations d'immigrés comme pour leurs enfants, une possibilité de démarche commune et adaptée sur le volet santé. Cette démarche permet d'aborder ces différentes questions et d'apporter des pistes de solutions partagées.

\section{Conclusion}

Notre étude a permis de modifier en partie l'environnement social et politique, en favorisant un repérage collectif des problèmes et des potentialités qui associe la population, avec un impact santé. L'emploi d'une méthodologie, testée et mesurée, permet un véritable terrain de réflexion sur l'interculturalité et la santé et donne un résultat satisfaisant sur l'évaluation de la démarche comme sur l'implication de la communauté en promotion de la santé. Cela confirme ainsi notre hypothèse d'amélioration des compétences sanitaires par cette démarche. Les évaluations mettent en évidence la pertinence d'une méthodologie qui permet non seulement la réussite du pilotage de cette démarche mais surtout l'impact positif d'intention sinon de changement de comportements individuels et communautaires sur l'éducation et la promotion de la santé pour eux et leur entourage. Ces résultats ont permis d'identifier des pistes d'intervention telles que la poursuite de la DCS pour des personnes issues de communautés, de tenir compte des croyances interculturelles, ou encore d'améliorer la communication sur la santé communautaire et la promotion de la santé.

Ce travail permet de documenter les croyances d'un échantillon de populations communautaires afin d'améliorer les interventions en santé et favoriser le bien-vivre en agissant sur les inégalités de santé.

\section{Références bibliographiques}

Albert O. Se former à l'interculturel : expériences et propositions ECLM. 2000;117.

Archinard C, Berthod M. Les compétences transculturelles dans le cadre de l'ESI (Doctoral dissertation, Haute école de santé Genève). 2014.

Ateba ASN. La «sociologie», une extra science de la santé et du salut. In : Pierre Meinrad Hebga: philosophie et anthropologie: actes du colloque international, 9-10 mars 2009, Université de Yaoundé I. Editions L'Harmattan. 2010:101.

Augoyard P, Renaud L. Le concept d' « empowerment » et son application dans quelques programmes de promotion de la santé. Promot Educ. 1998;5(2):28-35.

Baribeau, C., \& Germain, M. L'entretien de groupe: considérations théoriques et méthodologiques. Recherches qualitatives, (2010);29(1), 28-49.

Carde E. Les inégalités sociales dans l'accès aux soins: l'origine mise en catégories. Sociologie et 
sociétés. 2014;46(1):225-248.

Clanet C. L'interculturel. Introduction aux approches interculturelles en Education et en Sciences Humaines, Toulouse, Presses universitaire du Mirail. 1990:21

Collette, K., \& Rousseau, J. (2013). Littératie et responsabilité en santé. Globe: Revue internationale d'études québécoises, 16(1), 107-131.

Fainzang, S. La cure comme mythe: le traitement de la maladie et son idéologie à partir de quelques exemples ouest-africains. Cah Orstom (Sci Hum).1981;4:21-415.

Ferron C, Breton E. Recherche interventionnelle en Santé Publique : quand chercheurs et acteurs de terrain travaillent ensemble. La santé en action, Inpes. 2013;425.

Filiatrault J, Richard L. L'apport des théories des changements comportementaux aux interventions de prévention et de promotion de la santé de l'ergothérapeute. Canadian Journal of Occupational Therapy. 2005;72(1):45-56.

Gérard RM, Machteld A, Clemens H, et al. Preffi 2.0 : un outil néerlandais pour analyser l'efficacité des interventions en promotion de la santé. Promotion \& éducation. 2004:22-27.

Hall ET, Hacker AL. La danse de la vie: temps culturel, temps récu. Éditions du Seuil, DL. 1984.

Legros O, Olivera M. L'intermédiation sociale et interculturelle Réflexions libres à partir de terrains français et africains. Ville école intégration, Diversité. 2011;166:172-178.

Lessault D, Beauchemin C. Ni invasion, ni exode. Regards statistiques sur les migrations d'Afrique subsaharienne. Revue européenne des migrations internationales. 2009;25(1):163-194.

Marsicano E, Dray-Spira R, Lert F, Hamelin C. Les personnes vivant avec le VIH face aux discriminations en France métropolitaine. Institut National d'Études Démographiques (INED). 2014:516.

Marsicano E, Lydié N, Bajos N. Genre et migration: l'entrée dans la sexualité des migrants d'Afrique subsaharienne en France. Population, 2011;66(2):313-341.

O'Neill M. La Charte d'Ottawa: un manifeste pour «le manifestant». Global health promotion, 2012;19(2):67-69.

Reinberg O. Des Dieux et des Hommes: approches de la maladie en Afrique et en Europe. e-mémoires de l'Académie Nationale de Chirurgie. 2014;13(4):75-84.

Rootman I, Kaszap M, Frankish J. La littératie en santé: un concept en émergence. Promotion de la santé au Canada et au Québec : Perspectives critiques. 2006:87.

Stébé JM. La médiation sociale au cœur de la «crise urbaine». Informations sociales. 2012;2:82-88.

Tilmans-Cabiaux C, Mokrane S, Ravez L. Interculturalité et soins de santé : un questionnement éthique. Namur, BE: Presses universitaires de Namur. 2013.

Ting-Toomey S. Communicating across cultures. Guilford Press. 2012.

\section{Notes}

1. Selon la définition adoptée par le Haut Conseil à l'Intégration, le migrant appelé aussi l'immigré est une personne née étrangère à l'étranger et résidant en France. Les personnes nées françaises à l'étranger et vivant en France ne sont donc pas comptabilisées.

2. La Charte d'Ottawa pour la promotion de la santé a été établie à l'issue de la première Conférence internationale sur la promotion de la santé, Ottawa (Canada), du 17 au 21 novembre 1986. Elle énumère 5 axes dont l'axe 3 Renforcer l'action communautaire.

\section{Aucun conflit d'intérêt déclaré}

\section{Remerciements}

Cette étude est réalisée avec le soutien de la Fédération Régionale des Acteurs en Promotion de la Santé. Nous voudrions souligner l'indispensable participation de Philippe Fioyi Ayikon et Benny 
Vieira Batchi à la coordination. Nous remercions le comité de suivi de l'association BEC Bien-être communautaire de leur contribution. Nos sincères remerciements vont à l'ensemble des personnes qui ont donné un peu de leur temps bénévolement. Merci aux 16 membres de ce groupe de démarche communautaire en santé. 